\title{
Overcoming suppression in order to remember: Contributions from anterior cingulate and ventrolateral prefrontal cortex
}

\author{
Brice A. Kuhl, Itamar Kahn, Nicole M. Dudukovic, and Anthony D. Wagner \\ Stanford University, Stanford, California
}

\begin{abstract}
The ability to remember is often compromised by competition from irrelevant memories. However, acts of selective remembering can alter the competitive relationship between memories; memories that are selected against are weakened, whereas those that are retrieved are strengthened. Whereas the weakening of selectedagainst memories is typically evidenced by subsequently poorer recall of these memories, the present study tested the hypothesis that when previously selected-against memories can subsequently be successfully retrieved, such acts of successful retrieval are associated with engagement of neurobiological mechanisms that serve to detect and overcome competition. Consistent with this hypothesis, fMRI revealed that anterior cingulate cortex and right ventrolateral prefrontal cortex are differentially engaged during successful retrieval of previously selected-against memories, and that their engagement is directly related to the magnitude of weakening that is induced by prior acts of selecting against these memories.
\end{abstract}

Effective remembering requires the retrieval of goalrelevant memories in the face of competition from goalirrelevant memories (J. R. Anderson, 1974; M. C. Anderson, 2003; Shimamura, 2000). It is important that the very act of remembering changes the mnemonic competition experienced in the future, because retrieved memories are strengthened (Roediger \& Karpicke, 2006) and selectedagainst memories may be weakened or suppressed (M. C. Anderson, Bjork, \& Bjork, 1994; Levy \& Anderson, 2002). This reweighting of relevant and irrelevant memories is likely adaptive in that it reduces the competition experienced when the same memories are retrieved again (M. C. Anderson, 2003; Bjork, 1989; Kuhl, Dudukovic, Kahn, \& Wagner, 2007). However, when retrieval goals change and previously selected-against memories become relevant, the suppression that these memories suffered becomes an obstacle to retrieval, often resulting in an increased likelihood of forgetting (for a review, see Levy \& Anderson, 2002). When not forgotten, it has been hypothesized that the successful retrieval of previously selected-against memories places particularly high demands on control mechanisms that are supported by prefrontal cortex (PFC; Norman, Newman, \& Detre, 2007).

Behaviorally, the costs associated with selective retrieval have been most extensively studied using the retrieval practice paradigm (M. C. Anderson et al., 1994). In a standard variant of the paradigm, a series of cue-associate word pairs are initially studied, with multiple associates of each cue. Next, participants engage in retrieval practice, selectively retrieving some of the studied associates of some of the cues. Critically, this retrieval practice phase divides items into three categories: (1) practiced associates $(\mathrm{RP}+)$; (2) unpracticed associates of unpracticed cues, which serve as noncompeting, baseline items (NRP); and (3) unpracticed associates of practiced cues, which serve as selected-against competitors (RP-). Finally, memory for all cue-associate pairs is probed. Not surprisingly, on this final test, practiced items (RP+) are better remembered than unpracticed, baseline items (NRP), reflecting a benefit of retrieval practice. In contrast, unpracticed items that are selected against during retrieval practice $(\mathrm{RP}-)$ tend to be more poorly remembered than unpracticed items that do not compete during retrieval practice (NRP) - a phenomenon termed retrieval-induced forgetting that reflects a cost of selective retrieval.

The retrieval practice paradigm holds particular appeal for the effort to understand the behavioral consequences of selective retrieval because it allows for separate measurement of the facilitation of practiced (RP + ) items and the suppression of selected-against ( $\mathrm{RP}-$ ) items, with both measures expressed relative to baseline (NRP) recall. Similarly, the retrieval practice paradigm is well suited for framing an understanding of the neurobiological mechanisms that govern competitive retrieval. That is, although the cost that suppression carries is typically expressed in terms of the forgetting of RP- items, it is generally the case that many RP - items are nonetheless retrieved, putatively representing situations in which suppression has been successfully overcome. Thus, insight into the PFC control mechanisms that govern competitive retrieval, 
and that specifically allow for previously selected-against memories to be retrieved, can potentially be gained by comparing PFC engagement across retrieval conditions in the retrieval practice paradigm.

Although the PFC control mechanisms associated with overcoming retrieval-induced suppression have not, to our knowledge, been previously documented, several relevant lines of research inform predictions regarding the specific mechanistic components and corresponding PFC subregions that are likely to be implicated. As described below, our predictions focus on (1) anterior cingulate cortexmediated detection of competition, and (2) ventrolateral PFC-mediated selection and/or inhibition.

\section{PREFRONTAL CORTEX AND MNEMONIC COMPETITION}

Substantial evidence indicates that anterior cingulate cortex (ACC) plays a fundamental role in guiding behavior, although there is continued debate with regard to the specific mechanism through which this occurs. According to a prominent theory, ACC functions at an initial stage of control by detecting conflict between competing responses (e.g., Botvinick, Cohen, \& Carter, 2004; Kerns et al., 2004). Alternatively, it has been argued that ACC detects conditions in which an error is likely to occur, irrespective of whether conflict is experienced (Brown \& Braver, 2005). Finally, it has been argued that ACC is engaged when actions must be selected under conditions of uncertainty and, in such circumstances, integrates outcome information about the consequences of the selected actions (Walton, Devlin, \& Rushworth, 2004). Whereas the conflict-monitoring, error-likelihood, and action-outcome theories differ in the precise mechanistic framing of ACC function, it is important to note that they agree that ACC is responsive to situations in which response competition is present. Moreover, recruitment of ACC appears to extend beyond the domain of response competition (Van Veen \& Carter, 2002). Of particular interest, ACC activation has been observed when competition is present during retrieval from semantic memory (Van Veen \& Carter, 2005), working memory (Badre \& Wagner, 2004), and episodic memory (Bunge, Burrows, \& Wagner, 2004; Kuhl et al., 2007). Thus, evidence suggests that ACC responds to the presence of competition, including during mnemonic processing.

As argued by others, the detection of competition is thought to constitute an initial step in the implementation of cognitive control, with ACC computations serving to trigger engagement of control mechanisms supported by lateral PFC (Botvinick et al., 2004; Kerns et al., 2004). Given early evidence that ventrolateral PFC (VLPFC) may support the ability to overcome interference from irrelevant or competing representations (Iversen \& Mishkin, 1970), recent theorizing has focused on determining whether and how distinct anatomical subregions within left and right VLPFC support distinct forms of control that are engaged during mnemonic retrieval (e.g., Badre, Poldrack, Paré-Blagoev, Insler, \& Wagner, 2005; Dobbins \& Wagner, 2005; Gold et al., 2006).
Within left VLPFC, the inferior frontal pars triangularis subregion (which we term mid-VLPFC; Badre \& Wagner, 2007) has been repeatedly implicated in the selection between competing representations. For example, functional imaging studies have observed activation in this region when selection demands are high during retrieval from semantic memory (Badre et al., 2005; Thompson-Schill, D’Esposito, Aguirre, \& Farah, 1997), working memory (Jonides, Smith, Marshuetz, Koeppe, \& Reuter-Lorenz, 1998; Kostopoulos, Albanese, \& Petrides, 2007; Zhang, Feng, Fox, Gao, \& Tan, 2004), and episodic memory (Dobbins \& Wagner, 2005; Henson, Shallice, Josephs, \& Dolan, 2002; Sohn, Goode, Stenger, Carter, \& Anderson, 2003). Similarly, damage to or transient disruption of left mid-VLPFC is associated with performance deficits when mnemonic selection is required (Feredoes, Tononi, \& Postle, 2006; Thompson-Schill et al., 2002; Thompson-Schill et al., 1998).

While the contributions of left VLPFC subregions to mnemonic retrieval have been increasingly specified (Badre \& Wagner, 2007), the role of right VLPFC remains poorly understood. On the one hand, right VLPFC has frequently been implicated in episodic retrieval (for a review, see Fletcher \& Henson, 2001), but accounts of these activations have varied. It is interesting that right VLPFC is thought to play a critical role in attention, particularly in stimulus-driven orienting of attention (Corbetta \& Shulman, 2002; Weissman, Roberts, Visscher, \& Woldorff, 2006), suggesting that right VLPFC's contribution to retrieval is potentially described in terms of attentional orienting. Indeed, right VLPFC has been implicated in biasing attention toward visuoperceptual stimulus features during episodic retrieval (Dobbins \& Wagner, 2005), and right anterior VLPFC has been shown, within-participants, to be engaged during performance of both episodic retrieval and visual attention tasks (Cabeza et al., 2003).

With respect to the specific task of overcoming competition during retrieval, two additional lines of evidence are likely relevant. First, within the domain of cognitive control, it has been posited that right VLPFC supports the inhibition of prepotent, but irrelevant, responses (for a review, see Aron, Robbins, \& Poldrack, 2004). This view is supported by evidence that right inferior frontal damage (Aron, Fletcher, Bullmore, Sahakian, \& Robbins, 2003) or transient disruption (Chambers et al., 2006) impairs the ability to inhibit irrelevant responses. Moreover, it has been suggested that this form of inhibition may also operate within mnemonic contexts (Aron et al., 2004). Second, and most intriguing, a limited number of recent reports have implicated right VLPFC in situations that putatively require the inhibition or suppression of irrelevant episodic memories (M. C. Anderson et al., 2004; Depue, Curran, \& Banich, 2007; Kuhl et al., 2007; Wylie, Foxe, \& Taylor, 2008). ${ }^{1}$ Notably, however, these recent reports of mnemonic inhibition/suppression have tended to implicate right anterior VLFPC, or inferior frontal pars orbitalis, whereas the richer literature on response inhibition has tended to implicate right posterior VLPFC, or inferior frontal pars opercularis (Aron et al., 2004).

Taken together, extant data implicate both left and right VLPFC in overcoming competition from irrelevant mne- 
monic representations, with left mid-VLPFC putatively supporting selection between multiple competing representations, and right VLPFC supporting mechanisms that allow for selective attentional orienting and/or the inhibition of irrelevant, competing representations. Given these emerging hypotheses, we sought to further examine the functional responses of VLPFC subregions (specifically, left mid-VLPFC and right VLPFC) during competitive episodic retrieval.

To gain leverage on the PFC mechanisms engaged during competitive retrieval, we adapted the standard retrieval practice paradigm for use with functional magnetic resonance imaging (fMRI) in order to measure neural activity during retrieval of memories that were previously selected, as well as those that were previously selected against. Specifically, we evaluated fMRI data from the final test phase, separately considering neural responses associated with the retrieval of RP,$+ \mathrm{NRP}$, and RP-items. While retrieval-induced forgetting is typically evidenced by poorer recall success for RP-items, we hypothesized that differences in ACC and VLPFC engagement would be evident even across successful retrieval of RP,$+ \mathrm{NRP}$, and $\mathrm{RP}-$ items, reflecting differences in retrieval competition and selection/inhibition demands. Further, and of critical importance, we hypothesized that the differential engagement of ACC and VLPFC control mechanisms during RPretrieval would be a direct reaction to the suppression that these selected-against memories suffered, with these regions most robustly engaged when prior acts of mnemonic selection resulted in the greatest amount of suppression.

\section{Method}

Subjects. Twenty right-handed native English speakers (12 female, 18-32 years old) participated after having given informed consent in accordance with the institutional review board requirements at Stanford University. Data from one additional participant were excluded due to a failure to respond to more than $25 \%$ of the retrieval practice trials. Participants received $\$ 20 / \mathrm{h}$.

Materials and Procedure. The stimuli consisted of 40 cue words, each linked to 6 associate words, creating 240 cue-associate word pairs; all words were nouns. The pairs were divided into four subsets, each consisting of half of the associate words (3) of half of the cue words (20), creating 60 cue-associate pairs per subset. For each participant, one subset received retrieval practice.

The experiment contained four phases. During study, cueassociate pairs were intentionally encoded, with each cue studied with six associates ( $3 \mathrm{sec} /$ pair). During selective retrieval practice, participants were probed to covertly recall some of the studied associates of some of the cues - that is, one subset of cue-associate pairs. On each trial, a cue word was presented along with the first letter of a studied associate; participants had $3 \mathrm{sec}$ to indicate retrieval success or failure by keypress. Each practiced cue-associate pair was retrieved three times in this manner. Following a 15-min nonverbal filler task, a final test phase probed participants' memory for each of the studied associates, using the same probing procedure as during retrieval practice. The test-phase fMRI data are reported here (for further methodological details and a report of the retrieval practice fMRI data, see Kuhl et al., 2007).

fMRI procedures. Whole-brain functional imaging was conducted on a 3.0T GE Signa MRI system (GE Medical Systems), using a T2*-weighted 2D gradient echo spiral-in/out pulse sequence (Glover \& Law, 2001). The test phase consisted of two 360-volume functional runs $\left(\mathrm{TR}=2 \mathrm{sec}\right.$; $\mathrm{TE}=30 \mathrm{msec}$; flip angle $=70^{\circ}$; $3.125 \times 3.125 \times 5 \mathrm{~mm})$.
All image preprocessing and data analysis were performed using SPM2 (Wellcome Department of Cognitive Neurology, London). Functional data were corrected for slice-timing and head motion. Each participant's structural images were coregistered to their functional images and segmented into gray matter, white matter, and CSF. Gray-matter images were stripped of any remaining skull and then normalized to a gray-matter MNI template image. The normalized gray-matter image was used for normalization of the structural and functional images. Images were resampled to $3-\mathrm{mm}$ cubic voxels and smoothed with a Gaussian kernel ( $8 \mathrm{~mm}$ at full width, half maximum).

Data were analyzed under the assumptions of the general linear model (GLM). Trials were modeled as an event, using a canonical hemodynamic response function and its first-order temporal derivative. Correct and incorrect trials were modeled separately. The resulting functions were entered into a GLM with session treated as a covariate. Linear contrasts were used to obtain participant-specific estimates for each effect. These estimates were then entered into a second-level analysis, treating participant as a random effect, using a one-sample $t$ test against a contrast value of zero at each voxel.

Contrast maps were overlaid on a surface-rendered MNI canonical brain (SurfRend Toolbox; www.fil.ion.ucl.ac.uk/spm /ext/\#SurfRend). Conjunction analyses were performed by thresholding each contrast at $p<.032$ with an extent threshold of 5 voxels, yielding a joint Type I error of $p<.001$. For small-volume correction (svc), spherical volumes (10-mm radius) were generated for regions of a priori interest, centered at coordinates of previously reported activations (ACC, MNI coordinates -9, 36, 18; right VLPFC, 48, 27 , -6 ; Kuhl et al., 2007). Region of interest (ROI) analyses were performed on MarsBar-extracted (marsbar.sourceforge.net) data from all significant voxels within a $6-\mathrm{mm}$ radius of a maximum.

\section{Results}

Behavioral results. Performance at test revealed the benefits and costs of selective retrieval practice (Table 1). First, practiced items $(\mathrm{RP}+)$ were remembered better than baseline items (NRP) $[F(1,19)=90.85, p<.001]$. Second, selected-against, competing items (RP-) were less likely to be remembered than NRP items $[F(1,19)=13.52, p<$ $.005]$. This retrieval-induced forgetting is thought to reflect the suppression of competing memories caused by selective retrieval of target memories (M. C. Anderson \& Spellman, 1995). Test-phase reaction times (RTs) were slower for successfully retrieved RP - items than for NRP items $[F(1,19)=5.91, p<.05]$, which, in turn, were slower than for $\mathrm{RP}+$ items $[F(1,19)=99.06, p<.001]$.

fMRI results. Our hypotheses focus on the neural processes that are engaged in order to overcome competition during retrieval. It is important that, in the present study, the retrieval practice and test phases provided independent measures of retrieval competition. Specifically, during retrieval practice, changes in retrieval competition are thought to occur as a function of retrieval practice repetition. That is, previous work has demonstrated that the magnitude of retrieval-induced forgetting increases as a func-

Table 1

Test Phase Accuracy and Reaction Times (RT, in Milliseconds) by Condition

\begin{tabular}{cccccc}
\hline & \multicolumn{2}{c}{ Accuracy } & & \multicolumn{2}{c}{ RT } \\
\cline { 2 - 3 } \cline { 5 - 6 } Condition & $M$ & $S D$ & & $M$ & $S D$ \\
\hline RP+ & .479 & .167 & & 1,330 & 252 \\
NRP & .351 & .145 & & 1,693 & 255 \\
RP - & .302 & .137 & & 1,840 & 310 \\
\hline
\end{tabular}


tion of the number of retrieval practice attempts (Johnson \& Anderson, 2004; Levy, McVeigh, Marful, \& Anderson, 2007; Shivde \& Anderson, 2001). Thus, the strengthening of practiced items and the weakening of competing items that occur across retrieval practice attempts should reduce retrieval competition - a hypothesis supported both by models of interference (Mensink \& Raaijmakers, 1988) and by previously reported evidence that initial retrieval practice attempts are associated with greater PFC engagement than are subsequent retrieval practice attempts (Kuhl et al., 2007). During test, the strengthening of practiced items $(\mathrm{RP}+)$ and the weakening of competing items $(\mathrm{RP}-)$ should result in maximal retrieval competition when RPretrieval is compared with $\mathrm{RP}+$ retrieval.

To capitalize on the two independent measures of retrieval competition described above, we performed a conjunction analysis to identify regions that were jointly sensitive to competition at test and at retrieval practice. Inclusive masking of the successful $\mathrm{RP}->\mathrm{RP}+$ test-phase contrast by the contrast of 1 st $>3$ rd successful retrieval practice trials revealed activations in ACC/medial superior PFC, bilateral VLPFC, and bilateral posterior parietal cortex (see Table 2 and Figure 1). These results indicate a sensitivity of these regions to retrieval competition that is not specific to a single comparison; moreover, the results are consistent with our hypothesis that ACC and VLPFC are modulated by retrieval competition.

To more specifically interrogate ACC and VLPFC responses during test-phase retrieval, we next extracted the observed percent signal change from all suprathreshold voxels in three anatomically-defined regions of a priori interest: (1) ACC, (2) right VLPFC, and (3) left mid-VLPFC (anatomical automatic labeling; Tzourio-Mazoyer et al., 2002). Each region displayed a reliable condition $\times$ success interaction (see Figure 1) [ACC, $F(2,38)=4.45, p<$ .02 ; right VLPFC, $F(2,38)=7.89, p<.005$; left midVLPFC, $F(2,38)=5.55, p<.01]$, indicating that these regions were differentially engaged during retrieval of $\mathrm{RP}+, \mathrm{NRP}$, and RP - items. In ACC, the interaction was driven by marked activation during successful retrieval of RP - items, evidenced by (1) greater ACC activation during successful retrieval of RP - than of NRP items $[F(1,19)=6.37, p<.03]$; (2) an ACC success effect for $\mathrm{RP}-[F(1,19)=12.39, p<.005]$, but not for NRP or $\mathrm{RP}+$ items $(F \mathrm{~s}<1.34, p \mathrm{~s}>.25)$; and (3) a greater success effect for RP - than for NRP items $[F(1,19)=10.89$, $p<.005]$. By contrast, right VLPFC activation (1) did not differ between successful RP - and NRP retrieval $(F<1)$, and (2) demonstrated a retrieval success effect for both $\mathrm{RP}-[F(1,19)=8.61, p<.01]$ and $\mathrm{NRP}[F(1,19)=4.38$, $p<.05]$ items, but not for $\mathrm{RP}+[F(1,19)=1.22, p>.25]$ items. Finally, left mid-VLPFC activation did not differ between successful RP - and NRP retrieval $(F<1)$, nor did left mid-VLPFC display reliable success effects for any condition $(F \mathrm{~s}<2.18, p \mathrm{~s}>.15)$.

The preceding analyses indicate that ACC activation during retrieval marks a cost that suppression incurs for $\mathrm{RP}-$ items. On the other hand, while right VLPFC and left mid-VLPFC activation was modulated by retrieval demands, activation in these regions did not differ when
Table 2

Prefrontal and Parietal Cortical Regions Engaged by Retrieval Competition During I'est and During Retrieval Practice

\begin{tabular}{|c|c|c|c|c|}
\hline Region & $\sim \mathrm{BA}$ & $x$ & $y$ & $z$ \\
\hline \multicolumn{5}{|l|}{ Left Lateral PFC } \\
\hline Posterior VLPFC & $44 / 6 / 8$ & -42 & 6 & 39 \\
\hline Mid-VLPFC & 45 & -51 & 30 & 9 \\
\hline Mid-VLPFC & 45 & -54 & 36 & 12 \\
\hline Mid-VLPFC/DLPFC & $45 / 9$ & -39 & 21 & 27 \\
\hline Mid-VLPFC/DLPFC & $45 / 9$ & -48 & 24 & 24 \\
\hline Anterior insula/VLPFC & $13 / 47$ & -30 & 21 & 0 \\
\hline Anterior VLPFC & 47 & -51 & 42 & 3 \\
\hline Anterior VLPFC & 47 & -39 & 30 & -6 \\
\hline Anterior VLPFC & 47 & -33 & 33 & -3 \\
\hline Anterior VLPFC & 47 & -51 & 39 & -12 \\
\hline Frontopolar cortex & 10 & -48 & 51 & -3 \\
\hline DLPFC & 6 & -42 & 9 & 57 \\
\hline DLPFC & 6 & -36 & 6 & 57 \\
\hline \multicolumn{5}{|l|}{ Right Lateral PFC } \\
\hline Posterior VLPFC & $44 / 6 / 8$ & 45 & 6 & 33 \\
\hline Mid-VLPFC/DLPFC & $45 / 9$ & 45 & 24 & 21 \\
\hline Anterior VLPFC & 47 & 39 & 24 & -3 \\
\hline Anterior VLPFC & 47 & 39 & 21 & 3 \\
\hline DLPFC & $9 / 46$ & 57 & 33 & 24 \\
\hline \multicolumn{5}{|l|}{ Left Medial PFC } \\
\hline Medial SFG & 6 & -6 & 15 & 54 \\
\hline Medial SFG & 8 & -3 & 30 & 45 \\
\hline ACC & 24 & -6 & 6 & 27 \\
\hline \multicolumn{5}{|l|}{ Right Medial PFC } \\
\hline Medial SFG & 6 & 9 & 15 & 51 \\
\hline $\mathrm{ACC}$ & 24 & 6 & 9 & 27 \\
\hline $\mathrm{ACC}$ & $24 / 32$ & 12 & 27 & 27 \\
\hline $\mathrm{ACC}$ & 32 & 9 & 30 & 33 \\
\hline \multicolumn{5}{|l|}{ Left Parietal Cortex } \\
\hline ANG & 39 & -33 & -75 & 30 \\
\hline IPL & 40 & -42 & -42 & 45 \\
\hline IPS & 7 & -27 & -66 & 33 \\
\hline SPL/IPS & 7 & -33 & -66 & 45 \\
\hline SPL & 7 & -30 & -75 & 48 \\
\hline \multicolumn{5}{|l|}{ Right Parietal Cortex } \\
\hline SPL/IPS & 7 & 27 & -63 & 51 \\
\hline SPL & 7 & 30 & -78 & 48 \\
\hline
\end{tabular}

Note- BA, approximate Brodmann areas; $x, y$, and $z$ indicate location of local maxima in MNI coordinates; all local maxima $\geq 6 \mathrm{~mm}$ apart. VLPFC, ventrolateral prefrontal cortex; DLPFC, dorsolateral prefrontal cortex; SFG, superior frontal gyrus; ACC, anterior cingulate cortex; ANG, angular gyrus; IPL, inferior parietal lobule; SPL, superior parietal lobule; IPS, intraparietal sulcus.

comparing successful RP - with NRP retrieval. However, a striking aspect of participants' behavior was the considerable variability in the magnitude of $\mathrm{RP}-$ suppression. Thus, we next sought to test whether ACC and VLPFC subregions were modulated during $\mathrm{RP}-$ retrieval to the extent that RP - items were actually suppressed. Accordingly, for each participant, a suppression score was computedtest phase accuracy expressed as (NRP - RP-)/NRP (see Kuhl et al., 2007) - yielding a participant-specific measure of RP - suppression relative to baseline recall (range from $-21 \%$ to $51 \%$ ). This suppression score was then regressed upon the contrast of successful RP - to NRP retrieval, thresholded at $p<.005$, voxel extent $=5$, svc for a priori targeted ACC and VLPFC regions. This regression revealed three PFC foci: left ACC $\left(p_{\mathrm{svc}}<.05\right)$; right anterior VLPFC or inferior frontal pars orbitalis $\left(p_{\mathrm{svc}}<.06\right)$; and right superior frontal gyrus (see Table 3 ) - wherein 
A
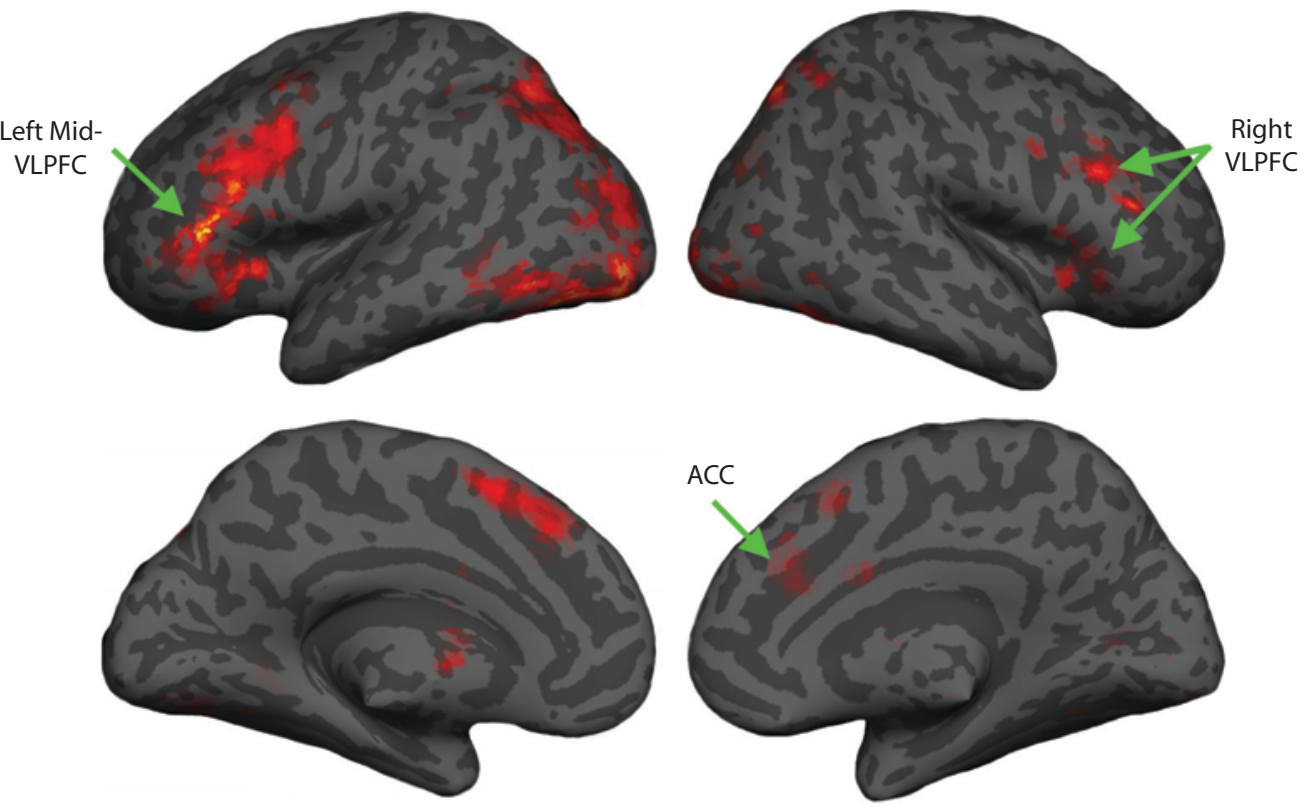

B

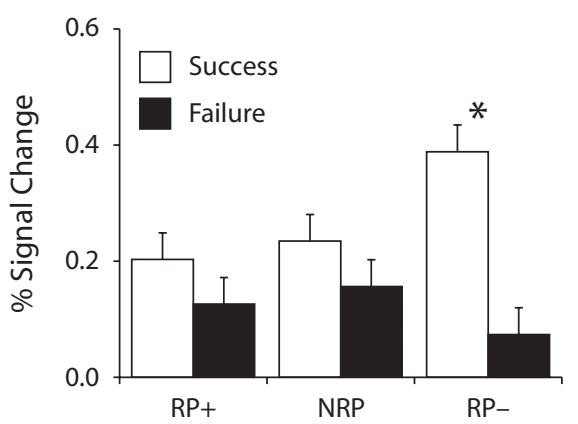

Left Mid-VLPFC

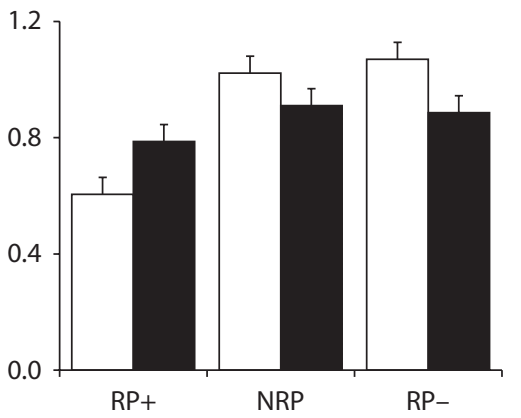

Right VLPFC

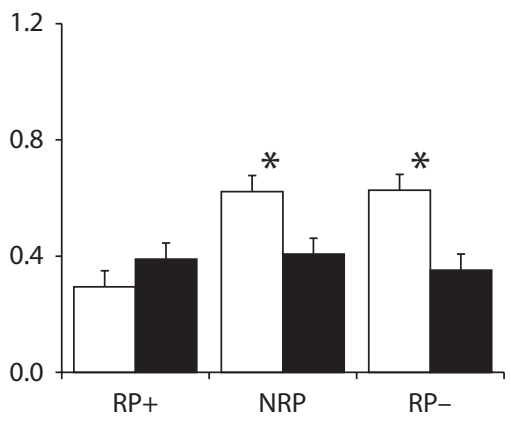

Figure 1. Regions modulated by retrieval competition during both retrieval practice and test. (A) Conjunction analysis of competition during retrieval practice (1st $>3$ rd retrieval practice) and during successful retrieval at test ( $R P->R P+$ retrieval). (B) Percent signal change for test-phase retrieval success and failure trials, for all suprathreshold bilateral ACC, left mid-VLPFC, and right VLPFC voxels from the conjunction analysis. These right VLPFC data are drawn from voxels spanning anterior, mid-, and posterior VLPFC; selective consideration of the data from right anterior VLPFC revealed a pattern that resembled that in ACC.

greater suppression of selected-against $(\mathrm{RP}-)$ memories was associated with greater activation when these memories were successfully retrieved (see Figure 2). ${ }^{2}$

That ACC and right anterior VLPFC were engaged to overcome suppression at test accords with our previous finding that these regions are disengaged during retrieval practice to the extent that suppression of competing memories occurs. Indeed, when the test-phase regression analysis described above was inclusively masked by the previously reported analysis, in which we regressed suppression score upon the contrast of $1 \mathrm{st}>3 \mathrm{rd}$ retrieval practice trials (Kuhl et al., 2007), the only observed foci in either PFC or parietal cortex were bilateral ACC and right anterior VLPFC (see Figure 2). Thus, across retrieval practice and test, ACC and right anterior VLPFC were sensitive to the strength of $\mathrm{RP}-$ items, with this sensitivity taking the form of reduced demands on these regions during retrieval practice of RP+ items and increased demands on these same regions during test-phase recall of RP- items.
Notably, in contrast with ACC and right anterior VLPFC, left mid-VLPFC activation did not reflect the costs associated with RP- suppression. Direct interrogation of left mid-VLPFC ${ }^{3}$ confirmed the absence of a relationship between suppression magnitude and activation during successful retrieval of RP - versus NRP items $(r=.02)$. This observation is somewhat surprising given (1) the extensive literature implicating left mid-VLPFC in selecting between competing representations (for a review, see Badre \& Wagner, 2007), and (2) the present demonstration that left mid-VLPFC activation was robustly modulated by retrieval competition (see Figure 1). As we discuss below, one possibility is that left mid-VLPFC and right anterior VLPFC resolve competition at functionally dissociable levels.

Other aspects of the present data further suggest a functional dissociation between left mid-VLPFC and right anterior VLPFC. First, a region (left mid-VLPFC, right anterior VLPFC, ACC) $\times$ condition $(\mathrm{RP}+, \mathrm{NRP}, \mathrm{RP}-$ ) 
Table 3

Regions in Prefrontal Cortex in Which the Magnitude of the Activation Difference Between RP - Recall and NRP Recall (RP $->$ NRP) Was Correlated with the Suppression Score

\begin{tabular}{lcccc}
\hline \multicolumn{1}{c}{ Region } & $\sim \mathrm{BA}$ & $x$ & $y$ & \multicolumn{1}{c}{$z$} \\
\hline ACC & 32 & -9 & 33 & 21 \\
Right superior frontal & 9 & 15 & 54 & 30 \\
Right anterior VLPFC & 47 & 48 & 30 & -12 \\
\hline
\end{tabular}

Note $-\sim \mathrm{BA}$, approximate Brodmann areas; $x, y$, and $z$ indicate location of peak activation in MNI coordinates; all local maxima $\geq 6 \mathrm{~mm}$ apart. ACC, anterior cingulate cortex; VLPFC, ventrolateral prefrontal cortex.

interaction $[F(4,76)=5.68, p<.001]$ revealed that left mid-VLPFC demonstrated less activation during successful $\mathrm{RP}+$ than during NRP retrieval $[F(1,19)=23.39, p<$ .001], which was not true of either right anterior VLPFC or ACC $(F \mathrm{~s}<1)$. Second, this left mid-VLPFC activation decrease during successful RP+ retrieval was positively correlated with the behavioral facilitation observed for $\mathrm{RP}+$ items, which was expressed as (RP+ - NRP)/NRP $(r=.54, p<.02) .{ }^{4}$ Thus, left mid-VLPFC was markedly less engaged when practiced, rather than nonpracticed, items were successfully retrieved at test, complementing reports that left mid-VLPFC is less active during repeated than during initial semantic retrieval (e.g., ThompsonSchill, D’Esposito, \& Kan, 1999; Wagner, Maril, \& Schacter, 2000). Moreover, left mid-VLPFC was disengaged during retrieval of practiced items to the extent that participants showed a behavioral benefit of practice (i.e., facilitated recall of $\mathrm{RP}+$ items).

Whereas left mid-VLPFC and right anterior VLPFC may support functionally dissociable forms of control, it has been suggested that ACC subserves a more general role in detecting representational conflict (Van Veen \& Carter, 2002). Accordingly, we tested whether ACC engagement covaried with the engagement of left mid-VLPFC, as well as of right anterior VLPFC. ACC activation correlated with both left mid-VLPFC and right anterior VLPFC engagement, but under different retrieval situations. Specifically, a multiple regression analysis indicated that during RP+ retrieval, the retrieval success effect in ACC was highly correlated with the success effect in left mid-VLPFC $(t=$ 4.66, $p<.001)$, but not with the success effect in right anterior VLPFC $(t<1)$ (see Table 4 and Figure 3$)$. In contrast, during RP - retrieval, the success effect in ACC was highly correlated with the success effect in right anterior VLPFC $(t=3.40, p<.005)$, but not with the success effect in left mid-VLPFC $(t<1)$. These data are consistent with the view that ACC subserves a domain-general role in detecting competition, triggering engagement of different VLPFC control mechanisms, depending on retrieval circumstances.

\section{Discussion}

The present data illustrate that successfully retrieving previously selected-against memories is accomplished through engagement of mechanisms within prefrontal cortex that govern competition - namely, ACC and right anterior VLPFC. Moreover, these regions were recruited in direct response to the suppression that selected-against memories suffered. In contrast, left mid-VLPFC was markedly less engaged when practiced, rather than unpracticed, associates were retrieved, but its engagement was not selectively associated with retrieving previously selected-against, suppressed memories. As outlined below, these data contribute to current theory regarding the contributions of ACC and VLPFC subregions during competitive retrieval.

ACC and mnemonic competition. The present data lend further support to the view that ACC serves to detect competition during episodic retrieval (Bunge et al., 2004; Kuhl et al., 2007), and, more generally, that ACC detects competition across representational levels (Braver, Barch, Gray, Molfese, \& Snyder, 2001; Van Veen \& Carter, 2002). Furthermore, within the context of episodic retrieval, we observed functional coupling between $\mathrm{ACC}$ and two distinct VLPFC subregions - right anterior VLPFC and left mid-VLPFC - but under different retrieval circumstances. Specifically, ACC coupled with left mid-VLPFC when previously selected memories were retrieved, whereas ACC coupled with right anterior VLPFC when previously selected-against memories were retrieved. The functional couplings between ACC and VLPFC are consistent with the theory that ACC serves to trigger lateral PFC control mechanisms (Botvinick et al., 2004; Kerns et al., 2004) and also complement prior reports that ACC functionally couples with lateral PFC during mnemonic processing (e.g., Badre \& Wagner, 2004; Bunge et al., 2004; Kuhl et al., 2007). Given ongoing debate concerning the precise mechanistic contributions of ACC to cognitive control, it is worth considering the present data with respect to the conflict-monitoring, error-likelihood, and action-outcome theories of ACC function.

According to conflict-monitoring theory (Botvinick et al., 2004), the fundamental contribution of ACC is to detect conflict between response possibilities. The present findings are well reconciled with conflict-monitoring theory, in that ACC was robustly engaged when retrieval involved selecting against prepotent, but irrelevant, memories (i.e., when RP - items were retrieved over RP+ items). Moreover, during retrieval of RP- items, ACC was differentially engaged to the extent that the participants suffered suppression of these items. However, it is also of note that ACC was more engaged during successful $\mathrm{RP}$ - retrieval than during failed $\mathrm{RP}-$ retrieval. This may be surprising, in that it could be argued that greater conflict should lead to greater retrieval failure. However, it seems equally plausible that the detection of conflict by ACC served to upregulate lateral PFC (e.g., right anterior VLPFC) control processes that enabled retrieval success. Thus, the conflict-monitoring model appears capable of accounting for the presently observed functional responses within ACC.

According to error-likelihood theory (Brown \& Braver, 2005), ACC signals conditions that have come to be associated with a high probability of committing an error, whether or not conflict is currently present or an error is ultimately committed. Error-likelihood theory in its standard form may have difficulty accounting for the present 
A

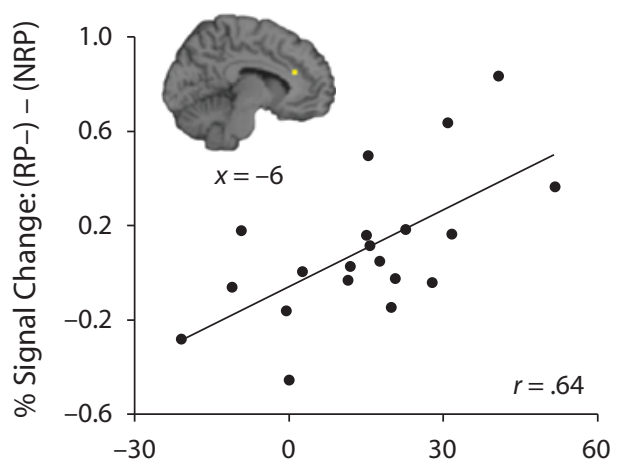

ACC
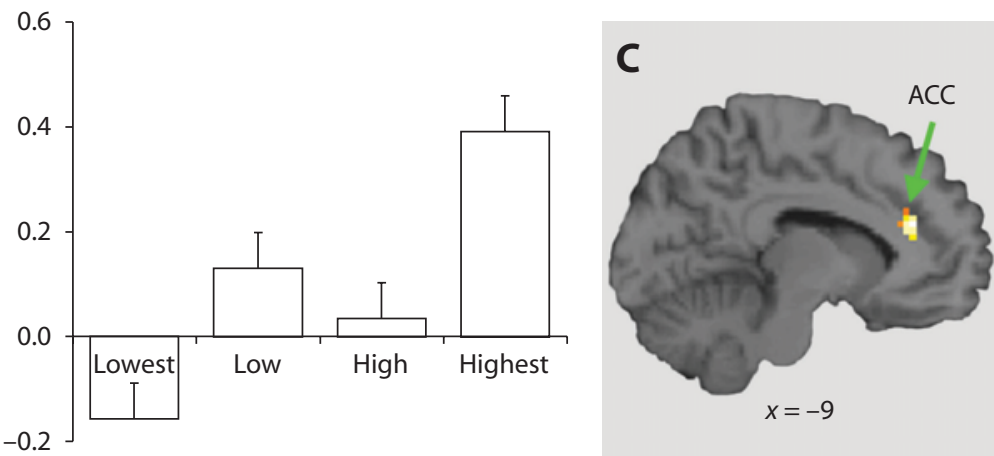

B Right Anterior VLPFC

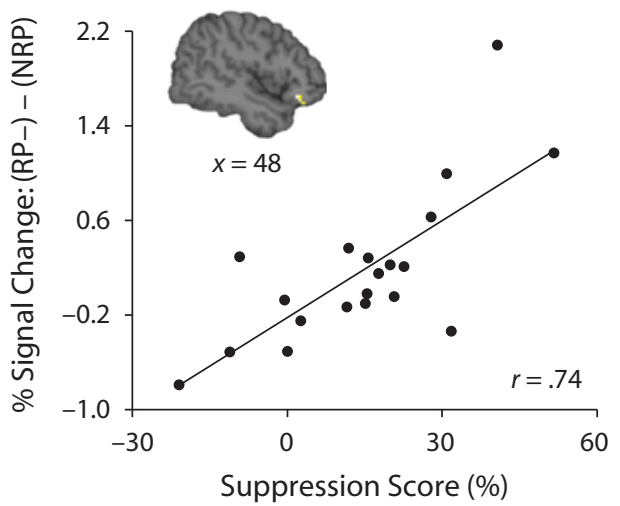

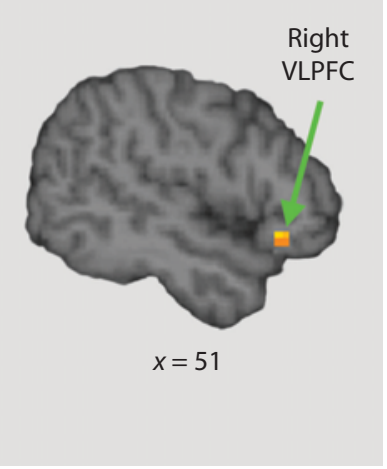

\section{Suppression Quartile}

Figure 2. Relationship of suppression score to PFC engagement during successful retrieval of RP - versus NRP items. (A) Betweenparticipants correlation (left) and the percent signal change difference separated by suppression score quartile (right) for left ACC; BA 32; MNI: $-9,33,21$. (B) Between-participants correlation and the percent signal change difference by quartile for right anterior VLPFC; BA 47; MNI: 48, 30, - 12. (C) Conjunction of retrieval-practice phase regression analysis (1st $>3$ rd retrieval, regressed upon suppression score) and test-phase regression analysis (RP $->$ NRP, regressed upon suppression score). PFC activations were limited to bilateral ACC ( BA 32; upper panel) and right anterior VLPFC ( BA 47; lower panel).

observation, that ACC was differentially engaged during $\mathrm{RP}$ - versus $\mathrm{RP}+$ retrieval, because $\mathrm{RP}$ - retrieval probes (i.e., the compound of the cue word plus the single letter of the target associate) were encountered for the first and only time during the test phase. Thus, the RP- retrieval probes had no prior association with error. Moreover, given that $\mathrm{RP}-$ retrieval probes were similar to $\mathrm{RP}+$ retrieval probes (i.e., they contained the same cue words), one might expect that the RP - retrieval cues would, if anything, elicit an association with retrieval success, not failure (error).

However, it might be argued that during retrieval practice, RP - items occasionally intrude during attempted retrieval of RP+ items, and, as a result, become tagged as errors. At test, the retrieval of RP - items might then elicit an association with error. According to this view, RP- trials should elicit ACC activation to the extent that the target $\mathrm{RP}$ - items are successfully retrieved - which is consistent with the observed data. It should be noted, though, that this error-tagging account would appear to represent a modified version of error-likelihood theory, as we understand it, because it would suggest that ACC engagement carries no functional consequence in the present context. That is, if successful RP - retrieval were to trigger ACC engagement, this would mean that ACC was engaged only after control was putatively required. Moreover, this modified version of error-likelihood theory cannot account for previously reported evidence that during the retrieval practice phase, ACC is most robustly engaged during initial retrieval practice attempts (Kuhl et al., 2007) — a situation in which neither the retrieval probes themselves nor the retrieval targets have associations with error. In contrast, conflict detection theory accounts for the engagement of ACC during initial retrieval practice attempts because these are situations of underdetermined, high-conflict retrieval.

Finally, it has been hypothesized that ACC serves to evaluate action-outcome relationships, supporting the selection of behavior when multiple response possibilities exist and supporting learning by integrating outcome information related to the consequences of selected actions (Walton et al., 2004). Whereas action-outcome theory is similar to conflict-monitoring theory in that it suggests that ACC will be engaged by situations in which underdetermined/ ambiguous responding occurs, action-outcome theory differs from conflict-monitoring theory in that it suggests that ACC also responds to positive and negative feedback, to the extent that this feedback is informative (Rushworth, Buckley, Behrens, Walton, \& Bannerman, 2007). 
Table 4

Multiple Regression Revealing the Relationship Between the ACC Success Effect and Left Mid-VLPFC and

Right Anterior VLPFC Success Effects Across Conditions

\begin{tabular}{clccc}
\hline Condition & \multicolumn{1}{c}{ Region } & $\beta$ & $t$ & $p$ \\
\hline \multirow{2}{*}{ RP+ } & Left mid-VLPFC & $.854^{*}$ & $4.662^{*}$ & $<.001^{*}$ \\
& Right anterior VLPFC & -.084 & -0.458 & .652 \\
NRP & Left mid-VLPFC* & $.442^{*}$ & $2.160^{*}$ & $.045^{*}$ \\
& Right anterior VLPFC & .296 & 1.451 & .165 \\
RP- & Left mid-VLPFC & .108 & 0.559 & .583 \\
& Right anterior VLPFC & $.655^{*}$ & $3.396^{*}$ & $.003^{*}$ \\
\hline
\end{tabular}

Note-ACC $=$ dependent variable; left mid-VLPFC and right anterior $\mathrm{VLPFC}=$ independent variables. $\quad$ *Statistically significant relationship with ACC $(p<.05)$.

In the present study, ACC was differentially engaged during $\mathrm{RP}-$ retrieval in relation to NRP or $\mathrm{RP}+$ retrieval. This finding is consistent with action-outcome theory because RP - retrieval represents a situation in which the appropriate response is weakly determined. This account also readily handles the correlation between the degree of $\mathrm{RP}$ - weakening (i.e., suppression) and ACC engagement during $\mathrm{RP}$ - retrieval. Moreover, while the present data do not directly test the prediction that $\mathrm{ACC}$ responds to feedback after uncertain responses, an intriguing, albeit speculative, possibility is that retrieval success itself may function as a form of feedback. Such a view would account for the greater ACC activity observed during successful RP - retrieval attempts. Finally, as with the conflict detection model, action-outcome theory provides an account for why ACC activity is greater during initial than during repeated retrieval practice - that is, with repeated retrieval practice, retrieval of target memories becomes more strongly determined.

Dissociable VLPFC mechanisms. Whereas both left mid-VLPFC and right anterior VLPFC have previously been implicated in governing competition during mnemonic processing, the present data point to a functional dissociation between these VLPFC subregions within the context of competitive episodic retrieval. We next consider the potential contributions of each of these VLPFC subregions within the present study, using extant theory to gain leverage on the mechanistic components that are required for retrieving previously selected-against memories.

A rich literature suggests that a core function of left mid-VLPFC is to select between competing, active representations across semantic, perceptual, and phonological domains (for a review, see Badre \& Wagner, 2007). Within the present study, left mid-VLPFC activation was reduced when participants retrieved previously selected $(\mathrm{RP}+)$ memories; moreover, this reduction was greater to the extent that participants showed a behavioral benefit of retrieval practice. Thus, the left mid-VLPFC activation reductions observed during $\mathrm{RP}+$ retrieval likely reflect reduced selection demands that accompany repeated retrieval (e.g., Thompson-Schill et al., 1999; Wagner et al., 2000). That is, as particular cue-associate pairs are practiced, and retrieval cues become more specifically associated with intended associates, the selection demands during retrieval decrease.
Whatever cost suppression carries for selected-against memories, this cost was not reflected in left mid-VLPFC activation; left mid-VLPFC was similarly engaged during NRP and RP - retrieval, irrespective of the magnitude of $\mathrm{RP}$ - suppression. This result is somewhat surprising, given evidence that left mid-VLPFC activation is driven by the potency of competing representations (Badre \& Wagner, 2007). However, whereas it was a priori hypothesized that RP - retrieval would engender higher selection demands than NRP retrieval, it is worth emphasizing that NRP retrieval itself involved selecting between six similar-strength associates, a situation that is comparable to the "high selection" conditions that have elicited robust left mid-VLPFC activation in the context of semantic retrieval (e.g., Badre et al., 2005; Thompson-Schill et al., 1997; Thompson-Schill et al., 1998). Thus, it is possible that left mid-VLPFC failed to demonstrate a significant difference in activation during NRP and RP - retrieval because this was a comparison between "high" and "higher" selection demands. Given that this outcome is a null result, we believe interpretative caution is warranted.

Although it is at present unclear why left mid-VLPFC, or inferior frontal pars triangularis, engagement was unrelated to RP - suppression, right anterior VLPFC, or inferior frontal pars orbitalis, engagement was clearly related to RP - suppression. Whereas left mid-VLPFC was highly sensitive to the practice-related facilitation of RP+ items, right anterior VLPFC did not show such a relationship. Complementing this dissociation, left mid-VLPFC was functionally coupled with ACC during successful RP+ and NRP retrieval, whereas right anterior VLPFC selectively coupled with ACC during successful RP - retrieval. Together, these data suggest that both left mid-VLPFC and

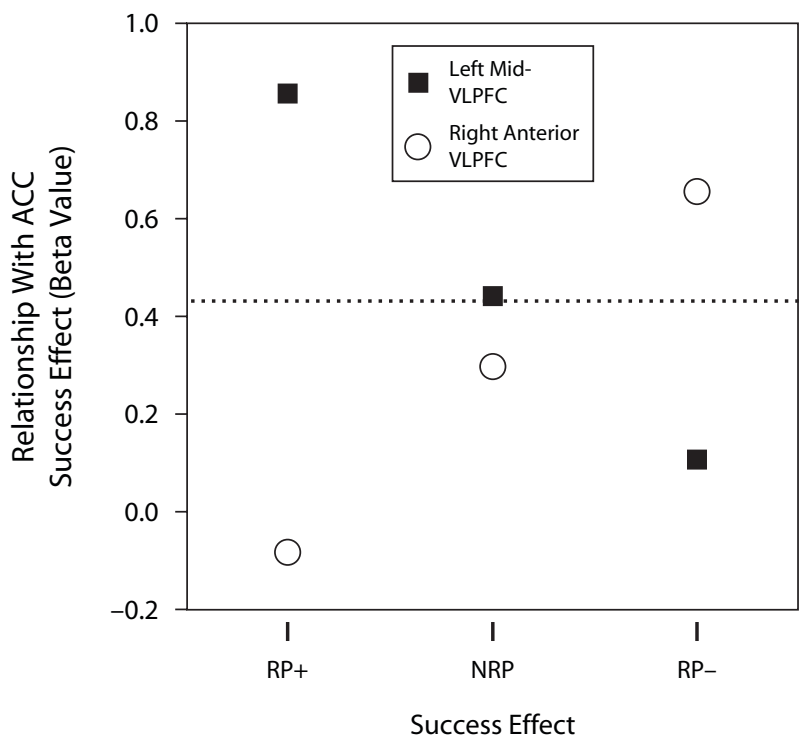

Figure 3. Beta values indicating the strength of the relationship between the success effect in ACC and left mid-VLPFC (square) and $A C C$ and right anterior VLFPC (circle), as a function of retrieval condition $(R P+, N R P, R P-)$. Dashed line reflects the threshold for statistical significance $(p<.05)$. 
right anterior VLPFC contribute to competitive retrieval and do so while functionally coupling with ACC, but that each may be differentially engaged, depending on particular retrieval circumstances (Dobbins \& Wagner, 2005).

Given the above argument, that right anterior VLPFC engagement reflects the contribution of a mechanism that appears distinct from left mid-VLPFC mediated selection, we next consider the potential mechanistic contributions of right anterior VLPFC. The present data are compatible with at least two accounts of right anterior VLPFC activation during competitive remembering: (1) selective orienting of attention toward retrieval probes/target memories, and/or (2) direct inhibition of prepotent, but irrelevant, memories. ${ }^{5}$

The demand for selective attentional orienting during $\mathrm{RP}-$ retrieval potentially takes two forms. First, the dominance of irrelevant RP+ items may have increased the demand to attend to specific perceptual properties of the RPretrieval probes in order to foster the ability of the probes to elicit successful retrieval of the target RP - items (Dobbins \& Wagner, 2005; Norman et al., 2007). Second, selective attention may have been engaged during RP - retrieval because the dominance of RP+ items resulted in a stimulusdriven orienting to nontarget representations (Corbetta \& Shulman, 2002). The latter possibility is particularly intriguing, given evidence that engagement of right anterior VLPFC has been associated with overcoming distraction during selective attention (Weissman et al., 2006). Thus, the present data accord well with an account of right anterior VLPFC based on the recruitment of selective attention to overcome dominant, irrelevant memories.

Although the present right anterior VLPFC data are highly compatible with a selective attention account, consideration of an inhibitory account is also warranted, given (1) substantial evidence that supports the hypothesis that right VLPFC mediates response inhibition (Aron et al., 2004), and (2) recent evidence that right anterior VLPFC is engaged in situations that putatively require mnemonic inhibition/suppression (M. C. Anderson et al., 2004; Depue et al., 2007; Kuhl et al., 2007; Wylie et al., 2008). Although our retrieval task required participants to make overt responses during the test phase, and thus clearly contained a response component, there are three arguments against a response inhibition account of the present right anterior VLPFC data. First, as described previously, response inhibition has been associated with right posterior VLPFC (Aron et al., 2004), whereas the present data implicate right anterior VLPFC. Second, the engagement of right anterior VLPFC during RP - retrieval was not correlated with RT measures, arguing against a direct relationship between right anterior VLPFC and overt responses. Third, others have reported right anterior VLPFC engagement associated with putative mnemonic inhibition in situations in which no overt response was being withheld (M. C. Anderson et al., 2004; Depue et al., 2007; Wylie et al., 2008).

As an alternative to response inhibition, a second possibility is that right anterior VLPFC (in the present study and related studies) reflects a form of inhibition that operates within the mnemonic domain and is analogous to the response inhibition supported by right posterior VLPFC. Although this is theoretically possible, the mnemonic inhibition account is difficult to separate from the selective attention account - at least within the context of the present study. For example, support for an inhibitory mechanism might come from evidence that right anterior VLPFC engagement carries consequences for the selected-against representations - which, during $\mathrm{RP}-$ retrieval, would be $\mathrm{RP}+$ items. Others have linked right anterior VLPFC engagement with mnemonic consequences for selected-against memories (Kuhl et al., 2007; Wylie et al., 2008), but because we did not have a second retrieval test exploring the consequences of RP - retrieval on subsequent RP + retrieval, the present data cannot address such a relationship. Moreover, we believe it is worth emphasizing that, to the extent that inhibition is a functional consequence of selective attention (e.g., Houghton \& Tipper, 1994; Miller \& Cohen, 2001), these alternatives may effectively reduce to a common account.

Whereas the present data do not support a response inhibition account of right anterior VLPFC engagement, they are consistent with either a selective attention account or a mnemonic inhibition account. Future work is needed to further specify the mechanistic contribution of right anterior VLPFC in situations in which mnemonic suppression/inhibition is putatively required. Moreover, although we point to a functional dissociation between left mid-VLPFC and right anterior VLPFC, the extent to which these mechanisms interact, and the nature of their interaction, remain questions for future study.

\section{CONCLUSIONS}

In summary, the present data reveal that when previously selected-against memories subsequently become relevant, successful retrieval of these memories is associated with engagement of ACC and multiple VLPFC subregions, reflecting distinct forms of control (Dobbins \& Wagner, 2005). Notably, ACC and right anterior VLPFC were selectively engaged during retrieval of previously selectedagainst memories to overcome the consequences of suppression. These data indicate that the processing resources that are conserved when selected-against memories are suppressed (Kuhl et al., 2007) are the same resources that become increasingly necessary when these memories later become relevant. Together, these data illustrate that the reweighting of memories that occurs as a result of selective retrieval affects not only what is ultimately remembered and forgotten, but also the extent to which later remembering relies on PFC-mediated control mechanisms. As such, these data contribute to our understanding of how we remember what we might otherwise forget.

\section{AUTHOR NOTE}

This study was supported by NIMH Grants R01-MH076932 and R01-MH080309 and the Alfred P. Sloan Foundation. Correspondence concerning this article should be addressed to B. A. Kuhl, Department of Psychology, Jordan Hall, Bldg. 420, Stanford, CA 94305-2130 (e-mail: bkuhl@stanford.edu).

\section{REFERENCES}

ANDERson, J. R. (1974). Retrieval of propositional information from long-term memory. Cognitive Psychology, 6, 451-474. 
ANDERson, M. C. (2003). Rethinking interference theory: Executive control and the mechanisms of forgetting. Journal of Memory \& Language, 49, 415-445.

Anderson, M. C., BJork, R. A., \& BJork, E. L. (1994). Remembering can cause forgetting: Retrieval dynamics in long-term memory. Journal of Experimental Psychology: Learning, Memory, \& Cognition, 20, 1063-1087.

Anderson, M. C., Ochsner, K. N., Kuhl, B., Cooper, J., Robertson, E., Gabrieli, S. W., ET AL. (2004). Neural systems underlying the suppression of unwanted memories. Science, 303, 232-235.

Anderson, M. C., \& Spellman, B. A. (1995). On the status of inhibitory mechanisms in cognition: Memory retrieval as a model case. Psychological Review, 102, 68-100.

Aron, A. R., Fletcher, P. C., Bullmore, E. T., Sahakian, B. J., \& Robbins, T. W. (2003). Stop-signal inhibition disrupted by damage to right inferior frontal gyrus in humans. Nature Neuroscience, $\mathbf{6}$, 115-116.

Aron, A. R., Robbins, T. W., \& Poldrack, R. A. (2004). Inhibition and the right inferior frontal cortex. Trends in Cognitive Sciences, $\mathbf{8}$, 170-177.

Badre, D., Poldrack, R. A., Paré-Blagoev, E. J., Insler, R. Z., \& WAGNER, A. D. (2005). Dissociable controlled retrieval and generalized selection mechanisms in ventrolateral prefrontal cortex. Neuron, 47, 907-918.

BADRE, D., \& WAGNeR, A. D. (2004). Selection, integration, and conflict monitoring: Assessing the nature and generality of prefrontal cognitive control mechanisms. Neuron, 41, 473-487.

BAdre, D., \& WAgner, A. D. (2007). Left ventrolateral prefrontal cortex and the cognitive control of memory. Neuropsychologia, 45, 2883-2901.

BJORK, R. A. (1989). Retrieval inhibition as an adaptive mechanism in human memory. In H. L. Roediger III \& F. I. M. Craik (Eds.), Varieties of memory and consciousness: Essays in honour of Endel Tulving (pp. 309-330). Hillsdale, NJ: Erlbaum.

Botvinick, M. M., Cohen, J. D., \& Carter, C. S. (2004). Conflict monitoring and anterior cingulate cortex: An update. Trends in Cognitive Sciences, 8, 539-546.

Braver, T. S., Barch, D. M., Gray, J. R., Molfese, D. L., \& SNyder, A. (2001). Anterior cingulate cortex and response conflict: Effects of frequency, inhibition and errors. Cerebral Cortex, 11, 825-836.

Brown, J. W., \& BraVER, T. S. (2005). Learned predictions of error likelihood in the anterior cingulate cortex. Science, 307, 1118-1121.

Bunge, S. A., Burrows, B., \& Wagner, A. D. (2004). Prefrontal and hippocampal contributions to visual associative recognition: Interactions between cognitive control and episodic retrieval. Brain \& Cognition, 56, 141-152.

Cabeza, R., Dolcos, F., Prince, S. E., Rice, H. J., Weissman, D. H., \& NyBERG, L. (2003). Attention-related activity during episodic memory retrieval: A cross-function fMRI study. Neuropsychologia, 41, 390-399.

Chambers, C. D., Bellgrove, M. A., Stokes, M. G., Henderson, T. R., Garavan, H., Robertson, I. H., et al. (2006). Executive "brake failure" following deactivation of human frontal lobe. Journal of Cognitive Neuroscience, 18, 444-455.

Corbetta, M., \& Shulman, G. L. (2002). Control of goal-directed and stimulus-driven attention in the brain. Nature Reviews Neuroscience, 3, 201-215.

Depue, B. E., Curran, T., \& Banich, M. T. (2007). Prefrontal regions orchestrate suppression of emotional memories via a two-phase process. Science, 317, 215-219.

DobBins, I. G., \& WAGNer, A. D. (2005). Domain-general and domainsensitive prefrontal mechanisms for recollecting events and detecting novelty. Cerebral Cortex, 15, 1768-1778.

Feredoes, E., Tononi, G., \& Postle, B. R. (2006). Direct evidence for a prefrontal contribution to the control of proactive interference in verbal working memory. Proceedings of the National Academy of Sciences, 103, 19530-19534.

Fletcher, P. C., \& Henson, R. N. A. (2001). Frontal lobes and human memory: Insights from functional neuroimaging. Brain, 124, 849-881.

Glover, G. H., \& LaW, C. S. (2001). Spiral-in/out BOLD fMRI for increased SNR and reduced susceptibility artifacts. Magnetic Resonance in Medicine, 46, 515-522.
Gold, B. T., Balota, D. A., Jones, S. J., Powell, D. K., Smith, C. D., \& ANDERSEN, A. H. (2006). Dissociation of automatic and strategic lexical-semantics: Functional magnetic resonance imaging evidence for differing roles of multiple frontotemporal regions. Journal of Neuroscience, 26, 6523-6532.

Henson, R. N. A., Shallice, T., Josephs, O., \& Dolan, R. J. (2002). Functional magnetic resonance imaging of proactive interference during spoken cued recall. NeuroImage, 17, 543-558.

Houghton, G., \& Tipper, S. P. (1994). A model of inhibitory mechanisms in selective attention. In D. Dagenbach, \& T. Carr (Eds.), Inhibitory processes in attention, memory, and language (pp. 53-112). San Diego: Academic Press.

Iversen, S. D., \& Mishrin, M. (1970). Perseverative interference in monkeys following selective lesions of the inferior prefrontal convexity. Experimental Brain Research, 11, 376-386.

Johnson, S. K., \& Anderson, M. C. (2004). The role of inhibitory control in forgetting semantic knowledge. Psychological Science, 15, 448-453.

Jonides, J., Smith, E. E., Marshuetz, C., Koeppe, R. A., \& ReuterLoRENZ, P. A. (1998). Inhibition in verbal working memory revealed by brain activation. Proceedings of the National Academy of Sciences, 95, 8410-8413.

Kerns, J. G., Cohen, J. D., MacDonald, A. W., III, Cho, R. Y., Stenger, V. A., \& CARTER, C. S. (2004). Anterior cingulate conflict monitoring and adjustments in control. Science, 303, 1023-1026.

Kostopoulos, P., Albanese, M.-C., \& Petrides, M. (2007). Ventrolateral prefrontal cortex and tactile memory disambiguation in the human brain. Proceedings of the National Academy of Sciences, 104, 10223-10228

Kuhl, B. A., Dudukovic, N. M., Kahn, I., \& Wagner, A. D. (2007). Decreased demands on cognitive control reveal the neural processing benefits of forgetting. Nature Neuroscience, 10, 908-914.

LeVy, B. J., \& ANDERSON, M. C. (2002). Inhibitory processes and the control of memory retrieval. Trends in Cognitive Sciences, 6 , 299-305.

Levy, B. J., McVeigh, N. D., Marful, A., \& Anderson, M. C. (2007). Inhibiting your native language: The role of retrieval-induced forgetting during second-language acquisition. Psychological Science, 18, 29-34.

Mensink, G.-J., \& RaAijmakers, J. G. W. (1988). A model for interference and forgetting. Psychological Review, 95, 434-455.

Miller, E. K., \& Cohen, J. D. (2001). An integrative theory of prefrontal cortex function. Annual Review of Neuroscience, 24, 167-202.

Norman, K. A., Newman, E. L., \& Detre, G. (2007). A neural network model of retrieval-induced forgetting. Psychological Review, 114, 887-953.

Roediger, H. L., III, \& KARPICKE, J. D. (2006). Test-enhanced learning: Taking memory tests improves long-term retention. Psychological Science, 17, 249-255.

Rushworth, M. F. S., Buckley, M. J., Behrens, T. E. J., Walton, M. E., \& Bannerman, D. M. (2007). Functional organization of the medial frontal cortex. Current Opinion in Neurobiology, 17, 220-227.

Shimamura, A. (2000). The role of the prefrontal cortex in dynamic filtering. Psychobiology, 28, 207-218.

Shivde, G., \& ANDERson, M. C. (2001). The role of inhibition in meaning selection: Insights from retrieval-induced forgetting. In D. S. Gorfein (Ed.), On the consequences of meaning selection: Perspectives on resolving lexical ambiguity (pp. 175-190). Washington, DC: American Psychological Association.

Sohn, M. H., Goode, A., Stenger, V. A., Carter, C. S., \& Anderson, J. R. (2003). Competition and representation during memory retrieval: Roles of the prefrontal cortex and the posterior parietal cortex. Proceedings of the National Academy of Sciences, 100, 7412-7417.

Thompson-Schill, S. L., D'Esposito, M., Aguirre, G. K., \& Farah, M. J. (1997). Role of left inferior prefrontal cortex in retrieval of semantic knowledge: A reevaluation. Proceedings of the National Academy of Sciences, 94, 14792-14797.

Thompson-Schill, S. L., D'EsPosito, M., \& Kan, I. P. (1999). Effects of repetition and competition on activity in left prefrontal cortex during word generation. Neuron, 23, 513-522.

Thompson-Schill, S. L., Jonides, J., Marshuetz, C., Smith, E. E., D'Esposito, M., KAN, I. P., ET AL. (2002). Effects of frontal lobe dam- 
age on interference effects in working memory. Cognitive, Affective, \& Behavioral Neuroscience, 2, 109-120.

Thompson-Schill, S. L., Swick, D., Farah, M. J., D’Esposito, M., Kan, I. P., \& KNight, R. T. (1998). Verb generation in patients with focal frontal lesions: A neuropsychological test of neuroimaging findings. Proceedings of the National Academy of Sciences, 95, 15855-15860.

Tzourio-Mazoyer, N., Landeau, B., Papathanassiou, D., Crivello, F., Etard, O., Delcroix, N., ET AL. (2002). Automated anatomical labeling of activations in SPM using a macroscopic anatomical parcellation of the MNI MRI single-subject brain. NeuroImage, 15, 273-289.

VAN VeEn, V., \& CARTER, C. S. (2002). The anterior cingulate as a conflict monitor: fMRI and ERP studies. Physiology \& Behavior, 77, 477-482.

Van Veen, V., \& Carter, C. S. (2005). Separating semantic conflict and response conflict in the Stroop task: A functional MRI study. NeuroImage, 27, 497-504.

Wagner, A. D., Maril, A., \& Schacter, D. L. (2000). Interactions between forms of memory: When priming hinders new episodic learning. Journal of Cognitive Neuroscience, 12(Suppl. 2), 52-60.

Walton, M. E., Devlin, J. T., \& Rushworth, M. F. S. (2004). Interactions between decision making and performance monitoring within prefrontal cortex. Nature Neuroscience, 7, 1259-1265.

Weissman, D. H., Roberts, K. C., Visscher, K. M., \& WoldorfF, M. G. (2006). The neural bases of momentary lapses in attention. $\mathrm{Na}$ ture Neuroscience, 9, 971-978.

Wylie, G. R., FoXe, J. J., \& TAYLOR, T. L. (2008). Forgetting as an active process: An fMRI investigation of item-method-directed forgetting. Cerebral Cortex, 18, 670-682.

Zhang, J. X., FenG, C.-M., FoX, P. T., GaO, J.-H., \& Tan, L. H. (2004). Is left inferior frontal gyrus a general mechanism for selection? NeuroImage, 23, 596-603.

\section{NOTES}

1. It should be noted, however, that the engagement of dorsolateral prefrontal cortex has also been associated with the inhibition/suppression of episodic memories (M. C. Anderson et al., 2004; Depue et al., 2007).

2. These effects were not attributable to RT differences; neither ACC nor right VLPFC activation correlated with between-participants differences in test-phase RT for RP- versus NRP items ( $p$ s $>.5$ ). Moreover, the RT difference for RP - versus NRP items was not correlated with the suppression score $(p>.4)$.

3. Given that peak activation from the competition conjunction fell in left mid-VLPFC (see Figure 1), an ROI was created, centered at this peak (MNI: $-51,30,9)$. This ROI was used for all subsequent left midVLPFC ROI analyses.

4. This correlation was significant for neither ACC nor right anterior $\operatorname{VLPFC}(p s>.1)$.

5. It might be predicted that selective attention/inhibition demands during $\mathrm{RP}$ - retrieval would increase not only to the extent that RP - items were weakened (suppressed), but also to the extent that RP+ items were strengthened (facilitated). While RP + facilitation was not correlated with right anterior VLPFC engagement during $\mathrm{RP}-\operatorname{retrieval}(p>.3)$, it should be noted that all 20 participants showed positive $R P+$ facilitation, whereas not all participants showed positive RP - suppression ( 15 out of 20). Thus, the lack of a between-participants relationship between RP+ facilitation and right anterior VLPFC engagement during RP - retrieval is potentially due to the restricted range of facilitation scores, rendering the suppression score a more useful between-participants measure of changes in competition.

(Manuscript received September 18, 2007; revision accepted for publication January 18, 2008.) 